\title{
Comparison of Two Methods of Transfusion Therapy for Stroke Prevention in Sickle Cell
}

\author{
Jennifer Keates-Baleeiro", Avery Mixon, Manoo Bhakta and Meghann McManus
}

Division of Pediatric Hematology Oncology, University of Tennessee College of Medicine, Chattanooga

"Corresponding author: Jennifer Keates-Baleeiro, Division of Pediatric Hematology Oncology, University of Tennessee College of Medicine, Chattanooga, USA, Tel: 423-778-7289; E-mail: Jennifer.Keates@erlanger.org

Received date: July 25, 2018; Accepted date: August 27, 2018, Publication date: September 05, 2018

Copyright: $\odot 2018$ Baleeiro JK, et al. This is an open-access article distributed under the terms of the Creative Commons Attribution License, which permits unrestricted use, distribution, and reproduction in any medium, provided the original author and source are credited.

\begin{abstract}
Aim: Chronic blood transfusions are essential to the care of sickle cell patients at high risk for morbidity and mortality due to stroke. In our comprehensive sickle cell centre, we support chronic transfusion with rapid manual partial exchange transfusions (RMPET) using a single access central line port. We do not have a comprehensive adult sickle cell program at our hospital, but upon transition of patients to an adult transfusion clinic they would be provided simple transfusion (ST) instead of exchange. Since we have a smaller sickle cell population and do not have erythrocytapheresis capabilities, we investigated whether switching our patients to simple transfusion would be feasible without compromising their haemoglobin $\mathrm{S}$ quantification parameters or ferritin levels.
\end{abstract}

Objective: To study our patients on chronic transfusion support and compare the different modalities to better understand the effects from switching from RMPET to ST.

Method: We used a prospective observational cohort design to determine which method of transfusion was best for lowering haemoglobin $S$ quantification. A crossover design was used so that each patient acted as its own control. Nine subjects between the ages of 4 years to 23 years were included in the study of 10 chronically transfused patients. Eight of nine subjects had Sickle cell SS hemoglobinopathy and one had S Beta ${ }^{\circ}$ Thalassemia. We additionally surveyed patient preference for each transfusion modality and nursing time to perform the transfusion.

Results: A Wilcoxon signed-rank test determined that there was not a statistically significant median difference in $\mathrm{Hb} \mathrm{S}$ at the end of the transfusion, both methods achieving $\mathrm{Hb} \mathrm{S}<30 \%$. There was also no statistically significant median difference in Ferritin levels. Conclusion: Our centre has opted to change our transfusion practice to straight transfusion and not RMPET which is more time consuming. Patients were more satisfied with the straight transfusion method. Nursing staff was appreciative of the shorter transfusion times. Our results may assist other smaller programs choose to use straight transfusion rather than rapid manual partial exchange for their chronically transfused patients.

Keywords: Chronic transfusion therapy; Sickle cell disease; Stroke prevention

\section{Background}

Stroke occurs in $10 \%$ of Sickle Cell Disease (SCD) patients before the age of 20 Years. Current standard of care for secondary stroke prevention in patients with SCD is chronic red blood cell (RBC) transfusions. Stroke recurs in $\sim 60 \%$ of patients without chronic RBC therapy and in $\sim 20 \%$ of patients with chronic transfusion while maintaining a haemoglobin $S$ percentage of less than $30 \%[1,2]$. Indefinite transfusion therapy is practiced since discontinuation after short-term or long-term prophylactic transfusions leads to recurrent overt strokes and more CNS damage, even with transition to hydroxyurea [3]. Chronic transfusion also prevents initial strokes in high-risk patients identified by trans cranial Doppler ultrasound (TCD). The Stroke Prevention Study in Sickle Cell Disease (STOP) demonstrated a $92 \%$ stroke risk reduction among 63 of 130 children with abnormal TCD results [4]. Rates of stroke declined significantly since implementing routine TCD screening and primary prophylactic transfusion therapy [5-7]. The subsequent STOP 2 trial supports the use of chronic transfusion indefinitely because discontinuation resulted in an increased rate of abnormal TCD conversion and development of overt stroke. Discontinuation of transfusions on the STOP 2 trial was also associated with a higher occurrence of silent cerebral infarcts, documented in 3 of 37 patients $(8.1 \%)$ in the continued-transfusion group compared with 11 of $40(27.5 \%)$ in the transfusion-halted group [8]. More recent studies demonstrate that SCD patients are also at risk for silent cerebral infarcts. An association between worsening vasculopathy shown by magnetic resonance angiography and progressive overt and silent infarcts on magnetic resonance imaging has been found [9]. More aggressive magnetic resonance imaging screening may be indicated, and this could result in more patients with SCD being treated with chronic transfusion.

Common chronic transfusion modalities include ST or RMPET. The goal of therapy is to reduce the haemoglobin S level either by diluting the blood (ST) or by removing and replacing the blood with non-sickle haemoglobin (RMPET). To prevent further brain injury, the goal of transfusion therapy is to lower the haemoglobin S quantification to less than $30 \%$ on a routine basis, usually with a monthly transfusion procedure. There are many large centres that utilize 
erythrocytapheresis which is considered the preferable method if available.

We focused on the types of RBC exchange therapy currently utilized in our Infusion clinic at T.C. Thompson Children's Hospital at Erlanger. We serve over 200 families, with only ten patients requiring chronic transfusion therapy. We determined which transfusion method was best for each patient for achieving the hematologic parameters of lower haemoglobin $\mathrm{S}$ quantification. We measured the amount of nursing time for each procedure and which method is preferred by patients.

\section{Methods}

We utilized a prospective observational cohort design to examine our aims. Nine of ten chronically transfused patients were eligible for the study. Our inclusion criteria were patients between the ages of 3 and 25 years, diagnosed with haemoglobin SS or S Beta ${ }^{\circ}$ Thalassemia, already receiving chronic transfusion therapy for stroke prevention with a Lansky play score of $100 \%$ or if over 16 years of age a Karnofsky score of $100 \%$. Exclusion criteria were more than two strokes with a modified Rankin score $>3$, a diagnosis of haemoglobin SC disease or chronic transfusion therapy for priapism. One patient receiving chronic transfusion with 3 prior strokes was excluded from the study.

Due to the very small sample size, a crossover design study was conceived so that each patient served as its own control. After appropriate IRB approval and consent to study, the nine patients were randomly assigned by blinded envelope to receive either the RMPET or ST. A three-month washout period was used before data collection. During that period patients received the mode of transfusion to which they were randomized. After collection of data, a second washout period occurred post crossover before a second set of data was collected. Each patient received three transfusions of each type. Thus six months of straight transfusion, followed by six months of RMPET transfusion or vice versa dependent on the random assignment. The design schema is presented on Figure 1.

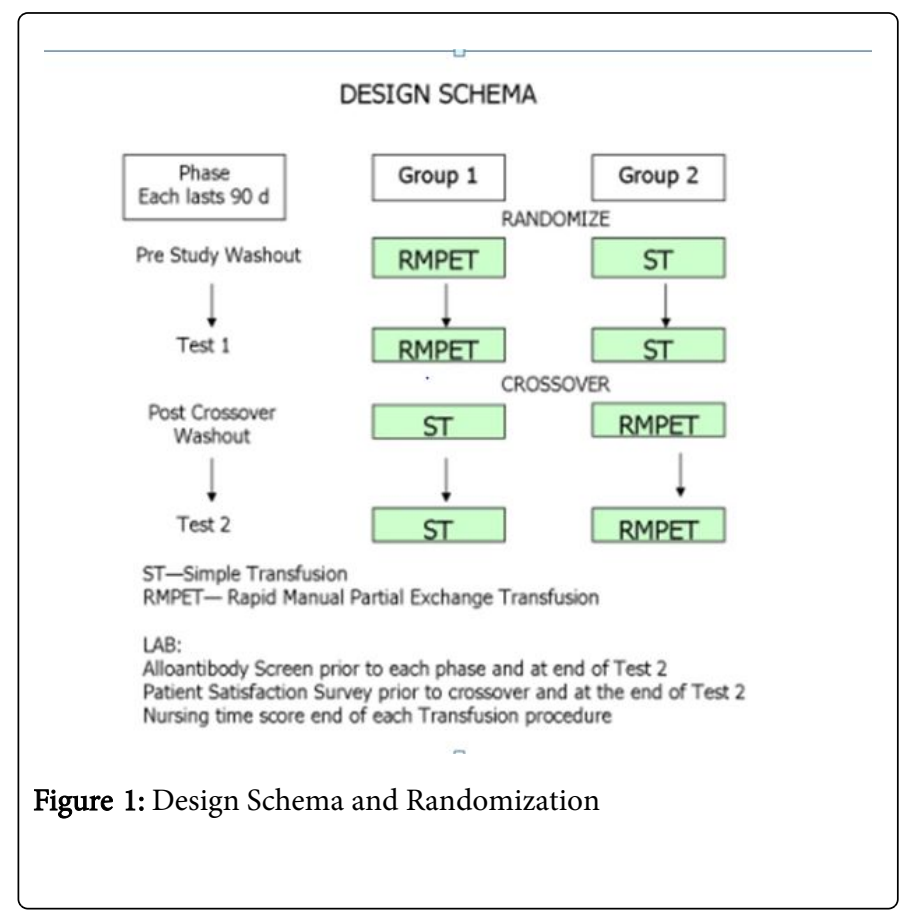

Data collection included dates of red blood cell transfusion, age of patient, gender, weight, height, Haemoglobin S quantification pre- and thirty minutes post transfusion, baseline haemoglobin/haematocrit, type of transfusion (RMPET or ST), blood volume, and presence of alloantibodies. Type and cross-match with extended antigen phenotype is done before transfusion. Time required by nursing to perform the RMPET was compared to the time needed for straight transfusion. We collected patient satisfaction surveys for each type of procedure performed.

The procedure for simple transfusion was to transfuse a volume of blood based on weight $(10-15 \mathrm{~mL} / \mathrm{kg})$. The procedure for rapid manual partial exchange transfusion (RMPET) was also weight based. For patients $<50 \mathrm{~kg}$, individual aliquots of blood removed and normal saline transfused was adjusted to $10 \mathrm{~mL} / \mathrm{kg}$. For patients weighing $>50$ $\mathrm{kg}, 500 \mathrm{~mL}$ of blood is removed, followed by $500 \mathrm{~mL}$ of normal saline, then a second aliquot $500 \mathrm{~mL}$ of blood is removed, and two units of PRBC are infused. If the patient has a large red blood cell mass, the above steps were repeated until three to four units of blood have been successfully transfused. There is a 30 -second wait time for equilibration to occur throughout the procedure with removal of blood from the patient or infusion of saline or packed red blood cells.

Care was taken post transfusion to evaluate for hyper viscosity and maintain haemoglobin between 10-12 gm/dL.

\section{Data Analysis}

Continuous variables were summarized by mean with standard deviation, median and $95 \%$ confidence intervals. Categorical variables were reported by frequency or proportions. Repeated measures ANOVA with covariates were used to compare the outcome variables between the two methods. Both comparisons within the group and between the groups were conducted with regard to hematologic parameters. Student's t-test was used to compare the nursing time between two methods. The patient satisfaction survey was taken for each type of transfusion. The responses ("very poor", "poor", "fair", "good" and "excellent") to questionnaires were transferred to 1 to 5 scales. A weighted score was calculated for each patient. For example, we calculated a weighted score based on five points for each person who said "excellent," four points for "very good," three points for "good" and so on. We then totalled the weighted responses for each question and averaged them to get the score. A Student's t-test or nonparametric method (Wallis rank test) was applied to compare the patient satisfaction score between the two methods to know which method was preferred by patients. Patient satisfaction surveys looked at satisfaction outcomes such as length of time for the transfusion method, time taken from school/work for preparatory lab work for transfusion and overall satisfaction with each method of transfusion was obtained.

\section{Results}

Patient characteristics are presented on Table 1. The Wilcoxon signed-rank test determined there was a statistically significant median difference in absolute haematocrit: The ST patients had a larger posttreatment haematocrit than the RMPET patients. The patients receiving ST also had a larger mean difference in haemoglobin compared to RMPET demonstrating that the pre and post haemoglobin levels were different depending on the method of transfusion. 
Page 3 of 4

A Wilcoxon signed-rank test determined that there was not a statistically significant median difference in Haemoglobin $S$ at the end of the transfusion, both methods achieving Haemoglobin $\mathrm{S}$ levels $<30 \%$. Indeed, patients receiving ST experienced a similar mean difference in their haemoglobin $S$ quantification compared to RMPET: ST (11.8667) to RMPET (11.5), z+0.652, $\mathrm{p}=0.515$.

A Wilcoxon signed-rank test determined that there was not a statistically significant median difference in Ferritin level. The ST patients experienced similar levels (2103.667) compared to RMPET patients (1956.00), $\mathrm{z}=-1.362, \mathrm{p}=0.173$. A Wilcoxon signed-rank test determined that there was no statistically significant median difference in Blood Volume levels either. The ST patients experienced similar levels (525.00) compared to RMPET patients (500.00), $\mathrm{z}=-0.674$, $\mathrm{p}=0.500$ ).

Not surprisingly, ST patients experienced significantly less transfusion time in minutes (96.6667) compared to RMPET patients (155.6667), $\mathrm{z}=2.666, \mathrm{p}=0.008$. There was not a statistically significant difference in nurse satisfaction between the two methods of transfusion; nurses experienced similar levels in ST (4.00) of satisfaction compared to RMPET nurses (4.00), $\mathrm{z}=-1.787, \mathrm{p}=0.074$. However, patient satisfaction favoured the straight transfusion modality.

\begin{tabular}{|l|l|l|l|}
\hline Subject & Age & Gender & Sickle cell type \\
\hline 1. & 9 years & Male & SS \\
\hline 2. & 20 years & Male & SS \\
\hline 3. & 8 years & Female & S Beta ${ }^{\circ}$ Thalassemia \\
\hline 4. & 18 years & Female & SS \\
\hline 5. & 10 years & Male & SS \\
\hline 6. & 15 years & Female & SS \\
\hline 7. & 23 years & Male & SS \\
\hline 8. & 4 years & Male & SS \\
\hline 9. & 21 years & Female & SS \\
\hline
\end{tabular}

Table 1: Study subject description and age at consent to study.

\section{Conclusion}

In our small sample of nine chronically transfused patients, there was not a statistically significant difference in the haemoglobin $S$ quantification at the end of the two transfusion methods using a crossover design methodology. At our Centre for Childhood Cancer and Blood Disorders we have opted to change our transfusion practice to straight transfusion and not RMPET which is more time consuming and does not achieve any greater difference in lowering haemoglobin $\mathrm{S}$ levels. Patients have more satisfaction with the straight transfusion method because it takes less time. While our nursing staff did not have a statistically significant result in the study for their satisfaction with either method, all were appreciative of the shorter transfusion times using straight transfusion (verbally reported).

We have continued to monitor our patients for a year post the conclusion of the study and have found our centre's adherence to straight transfusion and strict blood volumes continues to show haemoglobin S quantification post transfusion to hold at $<30 \%$. We are preparing to transition our oldest patients ( $>26$ years) to the adult ambulatory infusion centre who is only skilled in straight transfusion. Even though ferritin levels were not our primary outcome, there was no difference in those levels between the two groups and transfusion studies of longer duration have not found large differences $[10,11]$. We believe that strict adherence to an iron chelation regimen will enable our older patients to have success with the transition of care and hold their ferritin levels $<2500 \mathrm{ng} / \mathrm{dL}$.

Limitations in our study include the small number of patients, the length of time comparing the methods (one year of transfusions in total, six time periods of included data per patient) and a marked difference in how long some of our patients have received RMPET exclusively in our centre dating back to the year 1999. Another confounding factor is that all our patients are placed on iron chelation within the first eight transfusions they receive (deferasirox or, later in their chelation history, deferiprone) thus we do not have any chelationnaïve patients in this study unlike previous reported data on partial manual exchange [12]. Although we did not calculate serum iron accumulation per transfusion and previous studies have reported the superiority of erythrocytapheresis in reducing iron overload [11], the surrogate marker of ferritin in our study remained stable for the duration of our study. Future directions at our centre will be to investigate the liver iron concentration in our patients both by magnetic resonance imaging software and liver biopsy for chronic iron overload.

Chelation adherence remains a large barrier to delivering effective care in patients [13], but all of our patients maintained their adherence for the duration of this study. Although we cannot derive any conclusions about the effectiveness of chelation on our ferritin samples, we continue to advocate effective chelation therapy while receiving blood transfusions to prevent stroke.

In conclusion, we believe these results may help other similarly small programs serving the sickle cell population who need lifelong chronic transfusion therapy by providing support for continued straight transfusion instead of the more time-consuming RMPET.

\section{References}

1. Scothorn DJ, Price C, Schwartz D (2002) Risk of recurrent stroke in children with sickle cell disease receiving blood transfusion therapy for at least five years after initial stroke. J Pediatr 140: 348-354.

2. Hulbert ML, McKinstry RC, Lacey JL (2011) Silent cerebral infarcts occur despite regular blood transfusion therapy after first strokes in children with sickle cell disease. Blood 117: 772-779.

3. Ware RE, Helms RW (2012) Stroke with transfusions changing to hydroxyurea (SWiTCH). Blood 119: 3925-3932.

4. Adams RJ, McKie VC, Hsu L (1998) Prevention of a first stroke by transfusions in children with sickle cell anaemia and abnormal results on transcranial doppler ultrasonography. N Engl J Med 339: 5-11.

5. Eghan EH, Moore RH, Ichord R, Whitley SK, Kwiatkowski JL, et al. (2010) Transcranial doppler ultrasonography and prophylactic transfusion program is effective in preventing overt stroke in children with sickle cell disease. J Pediatr 157: 479-484.

6. McCarville MB, Goodin GS, Fortner G (2008) Evaluation of a comprehensive transcranial doppler screening program for children with sickle cell anemia. Pediatr Blood Cancer 50: 818-821.

7. McCavit TL, Xuan L, Zhang S, Flores G, Quinn CT, et al. (2013) National trends in incidence rates of hospitalization for stroke in children with sickle cell disease. Pediatr Blood Cancer 60: 823-827.

8. Adams RJ, Brambilla D (2005) Discontinuing prophylactic transfusions used to prevent stroke in sickle cell disease. N Engl J Med 353: 2769-2778. 
Citation: Baleeiro JK, Mixon A, Bhakta M, McManus M (2018) Comparison of Two Methods of Transfusion Therapy for Stroke Prevention in Sickle Cell. J Blood Disord Transfus 9: 405. doi:10.4172/2155-9864.1000405

Page 4 of 4

9. Casella JF, King AA, Barton B (2010) Design of the silent cerebral infarct transfusion (SIT) trial. Pediatr Hematol Oncol 27: 69-89.

10. Mian HS, Ward R, Telfer P (2015) Optimal manual exchange transfusion protocol for sickle cell disease: A retrospective comparison of two comprehensive care centers in the United Kingdom and Canada. Hemoglobin 39: 310-315.

11. Fasano RM, Leong T, Kaushal M, Sagiv E, Luban NLC, et al. (2012) Effectiveness of red blood cell exchange, partial manual exchange and simple transfusion concurrently with iron chelation therapy in reducing iron overload in chronically transfused sickle cell anemia patients. Transfusion 56: 1707-1715.

12. Savage WJ, Reddoch S, Wolfe J, Casella JF (2013) Partial manual exchange reduces iron accumulation during chronic red cell transfusions for sickle cell disease. J Pediatr Hematol Oncol 35: 434-436.

13. Aloni MN, Le PQ, Heijmans C, Huybrechts S, Devalck C, et al.( 2015) A pilot study of manual chronic partial exchange transfusion in children with sickle disease. Hematology 20: 284-288. 